\title{
DEVELOPMENT OF A CONVOLUTIONAL NEURAL NETWORK MODEL FOR BRAIN ABNORMALITY CLASSIFICATION
}

\author{
Hetal Barad \\ Research Scholar, Faculty of Computer Science and Applications, \\ Charotar University of Science and Technology, Changa, India. \\ hettal_barad8869@yahoo.com \\ Atul Patel \\ Dean and Professor, Faculty of Computer Science and Applications, \\ Charotar University of Science and Technology, Changa, India \\ atulpatel.mca@charusat.ac.in
}

\begin{abstract}
Medical image includes various image modalities and procedures, which helps in patient treatment and diagnosis. Many abnormalities are occurred in human brain. Among them, with the frequent occurrence of the tumor disease and its complexity, brain tumor has become important research topic in the medical field. The brain tumor diagnosis is mainly based on the medical imaging data analysis from the images of brain tumor. However, the accurate analysis tumor medical image is highly dependent on the knowledge, experience and eye strain of a doctor. with the advancement in the field of artificial intelligence along with computer vision provided opportunity for brain tumor identification more precisely without explicit feature extraction and human intervention. Deep Learning, the recent advancement in the field of artificial intelligence, makes system enable learning features automatically for the given problem without human expertise. In deep learning, convolutional neural network (CNN) is a promising architecture that applied to visual imagery. The aim of this paper is to develop and propose a CNN model for classifying brain abnormalities by considering one of the abnormality i.e. brain tumor. For that, the different benchmark CNN models are applied and evaluated. The results obtained from these models are analysed that motivates us to develop the custom CNN model for the said problem. From the performance evaluation, it is found that, the proposed model outperforms with good accuracy.
\end{abstract}

Keywords: Brain Abnormality; Deep learning; CNN; Multi-class Classification

\section{Introduction}

With advancement in the modern medical science standards, medical research and diagnosis became easy with the help of medical imaging technology. So, medical diagnostic images play an important role in the medical field research. Medical image includes various image modalities and procedures, which helps in patient treatment and diagnosis. Hence, it plays a dominant role in deciding the actions for the benefit of the patients' health. Many abnormalities are occurred in human brain. Among them, with the frequent occurrence of the tumor disease and its complexity, brain tumor has become important research topic in the medical field. Unusual growth of the tissues forms the tumor. It is an uncontrolled growth of cancer in the particular part of the body. There are various forms of the tumor with differentiable features and each demands special type of treatment [1]. The major categories of the brain tumor are primary brain tumor and metastatic brain tumor. Primary brain tumor mainly forms in the brain while the metastatic tumor spread via brain and forms elsewhere in the body. The brain tumor symptoms highly dependent on the type, size and location of the tumor[2]. It mostly occurs when circulation of the blood stuck throughout the brain because of the tumor. Common symptoms of the brain tumor are problem in walking and balancing, vomiting and headache. The brain tumor diagnosis is mainly based on the medical imaging data analysis from the images of brain tumor [3]. So, accurate analysis of the tumor image is the primary step in predicting condition of the tumor affected patient. However, the accurate analysis tumor medical image is highly dependent on the knowledge, experience and eye strain of a doctor. Generally, MRI - Magnetic Resonance Imaging is used for obtaining quality imaging. Hence, MRI is widely used in brain scanning images to identify brain tumor easily from it. Digital representation of the tissue features that can be obtained from the tissue plan can be obtained using MRI very easily. MRI scanner produced horizontally and vertically sliced images. 
Nervous system and brain cancer is mentioned as the 10th leading cause for the deaths worldwide [4]. The survival rate of the people with the brain cancer in last five years is the $34 \%$ for men and $36 \%$ women [5]. However, human interpretation of this may leads to false identification of brain tumor. Using computerized system for brain tumor identification, based on appearances of the basic symptoms from the image, make tumor identification easy and more precise. There are various technologies available with makes digital image processing feasible and allow to extract meaningful information out of it. Image processing using machine learning technology used decade ago for the various images related task like segmentation, classification, highlighting specific area of the image and many more. However, image processing demands manual feature extraction, which again requires domain expertise and makes it human perception dependent. However, with the advancement in the field of artificial intelligence along with computer vision provided opportunity for brain tumor identification more precisely without explicit feature extraction and human intervention. Deep Learning, the recent advancement in the field of artificial intelligence, makes system enable learning features automatically for the given problem without human expertise [6]. In deep learning, convolutional neural network $(\mathrm{CNN})$ is a promising architecture that applied to visual imagery [7].

The aim of this paper is to develop and propose a CNN model for classifying brain abnormalities by considering one of the abnormality i.e. brain tumor. For that, the different benchmark CNN models are applied and evaluated. The results obtained from these models are analysed that motivates us to develop the custom CNN model for the said problem.

\section{Survey of Literature}

Ming Li \& et al. [8] proposed three-dimensional MRI brain tumor identification model, which combines CNN with multimodal information fusion, called a Multi-CNN. This model collects the brain lesions with the different characteristics of the modal in three-dimensional space. To maintain the speed of the network and to avoid the problem of overfitting normalization layer was added between convolutional layer and pooling layer. The experimentation shows that compared with two-dimensional and single model brain tumor detection model, Multi-CNN modal provides significantly high accuracy. Authors in the paper [9], presented the brain tumor identification modal by segmenting MRI images using Fuzzy C-Means clustering and classification of the tumor was done using CNN modal. They have compared the results of various machine learning classification algorithms like SVM, Logistic Regression, K-nearest neighbor, Random Forest and Naïve Bayes, with CNN modal. Accuracy provided by CNN model was $97.42 \%$, which is highest, compared with other machine learning classifiers.

Hasan Ucuzal \& et al. [10] developed the web based brain tumor classification system using high-precision T1 MRI images and convolutional neural network. According to the performance matrices obtained, authors were able to achieve $98 \%$ of classification accuracy on training dataset and $91 \%$ accuracy on test dataset. The system was implemented on two datasets and obtained $96.13 \%$ and $98.70 \%$ accuracy respectively for both. Authors in the paper [11] proposed new CNN based method for automatic segmenting Glioma, Meningioma, and Pituitary types of brain tumors. Results of the network highlighted that separation of the images based on the angles improves the accuracy of the segmentation. Evaluation of the network shows that 0.73 Dice score was obtained for a single network and 0.79 obtained for the multiple networks. Ming Sihang Zhou \& et al. [12] presented high-resolution multi-scale encoder-decoder network (HMEDN) model to deal with the blurry medical image segmentation. To exploit the comprehensive semantic information multi-scale dense connections with encodedecoder structure was introduced. Experimentation on the network shows that along with the network complexity increasing the semantic feature maps improves the overall network performance largely. Authors in the paper [13] presented the network with 3 convolutional blocks, each with 11 layers depth and consists 6 convolutional layers with $3 \times 3$ filters, 2 max-pooling layers and three dense fully-connected layers. Authors have performed the preprocessing intensity equalization on all the images by normalizing and by computing standard deviation and mean intensity, values removed the noise form the images. Proposed model was able to achieve the $88 \%, 83 \%$ and $77 \%$ accuracy with the whole tumor, core tumor and active tumor in the BraTS dataset.

Salma X sun \& et al. [14] proposed a SegNet model for brain tumor segmentation. The specialty of the proposed model is that it separately train all modalities and combine the output at the post-processing stage of the SegNet. There are pair encoder and decoder in the architecture. Model contains 13 convolutional layers consists $3 \times 3$ filters, ReLU activation layer, batch normalization and $2 \times 2$ filter max-pooling layer. The segmentation technique was able to achieve accuracy of $85 \%$ for entire tumor, $81 \%$ for core and $79 \%$ for enhancing tumor. Authors in the paper [15] presented two pathway CNN model for tumor identification from the MRI image. The network extracts large path size $37 \times 37$ and minor 19x19 patch size at the same time. To avoid the problem of overfitting in the network dropout and maxout layers were used. Architecture consists six convolutional layers with varying filter size, which enables model to learn the different size features. The model achieved accuracy $80 \%$ with complete, $67 \%$ with core and $85 \%$ with enhancing tumor respectively. The literature shows that deep learning and CNN models can be applied for the brain tumor classification promptly. 


\subsection{Dataset Accumulation}

\section{Materials and Methods}

A benchmark dataset for Brain Tumor has been acquired from fighshare data repository. [16] It contains total of 3064 images of type T1-weighted contrast-enhanced images. They have collected these slices from 233 patients. The tumor types they considered are meningioma, glioma and pituitary tumor. There are 708 slices are available for meningioma tumor, 1426 slices are available for glioma tumor and 930 slices are available for pituitary tumor. The data are available matlab format with .mat extension. For conducting experiment, the dataset converted into .jpg files and arrange into appropriate class label folders. The following figure 1 represents the sample image of each class available in the dataset.

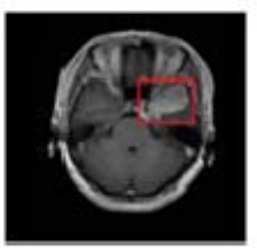

Meningioma

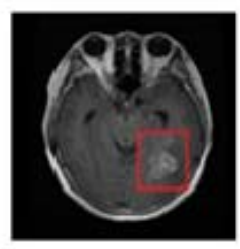

Glioma

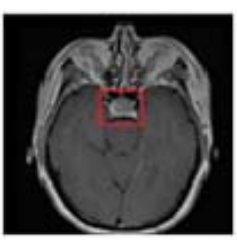

Pituitary

Fig. 1. Sample of Image of Each Class from the Dataset

\subsection{Data Augmentation}

Deep learning models normally required huge dataset to train the model. The performance is often improved with the amount of the data available. Moreover, the data should be in balanced and diverse form [17]. It contains the techniques that artificially increased the data size and diversity of the entire dataset. The common augmentation techniques are flipping, cropping and rotating the images. We have applied the said augmentation techniques to increase the dataset size from 3064 to 12067 images. After data augmentation, there are 3709 images available for meningioma, 4427 images available for glioma and 3931 images are available for pituitary tumor.

\subsection{CNN Network Architecture}

We have first implemented pre-trained benchmark CNN models trained on ImageNet dataset to evaluate the performance of the same. Motivating from the results obtained, we have developed our own CNN model for brain abnormality detection using the brain tumor dataset. Various state-of-the-art CNN models are experimented and evaluated by fine-tuning it for brain tumor detection and classification. The models evaluated include VGG16, VGG19, ResNet50V2, ResNet101V2, MobileNetV2 and DenseNet121 and proposed deep CNN model.

\subsection{Various Pre-Trained Benchmark CNN Models Used for Tumor Classification}

Deep learning is the key instrument in the artificial intelligence for the application like image classification [18]. The goal of image classification is to classify a specific image from set of possible categories [19]. Pre-trained models are already trained model on the benchmark dataset to solve a problem similar to various real time classification tasks. The ImageNet dataset consists 15 million high-resolution images categorized into around 22,000 used for training all the benchmark CNN models. The various pre-trained models are used in this research work for the brain tumor classification task. The model used was VGG16, winner of the ILSVR(Imagenet) competition in 2014. VGG16 consists convolutional layers of 3x3 filters and with stride 1 and used padding and max-pooling layers with $2 \times 2$ filters and stride 2 . At the end, it has two fully connected layers [20]. There is consistent arrangement of convolution and max-pooling layers throughout the network. The next model used is VGG19, which follows similar architecture like VGG16, except it has 19 layers whereas VGG16 has 16 layers. MobileNetV2 used for tumor classification follows inverted residual architecture. Unlike traditional residual models, input and output of the residual block are thin bottleneck blocks. MobileNetV2 consists two types of blocks one residual block with 1 stride and another with stride 2 for down sampling [21]. Another model used in the research work is ResNet - winner of the ImageNet challenge 2015. With the invention of ResNet, new era of training deep neural networks with 150+ layers were started [22]. Prior to that it was difficult to train deep neural network because of vanishing gradient problem. The basic idea behind the ResNet architecture was the identity shortcut connection, which fits the input from the previous layer to the subsequent layer without altering the input. ResNet50 is the smaller version of the ResNet architecture and frequently used as the starting point in transfer learning. ResNet101 is the 101 layers' deep convolutional neural network. The pre-trained version of the network train over more than million images from the ImageNet dataset was used for the brain tumor classification task for the comparison with other network. In 2017, paper representing DenseNet (Dense Convolutional Network) won the best research paper award with 2000 citations. 
DenseNet was able to achieve high accuracy compared with ResNet using fewer parameters. In this architecture, each layer of the network obtains additional input from all its previous layers and passes its own feature map to next subsequent layers of the network. Thus, each layer receives collective knowledge from its all preceding layers, which helps in improving the overall accuracy of model. DenseNet network become compact and thinner as each layer receives feature map from its all preceding layers. DenseNet121 with 121 layers used in this research to solve the problem of brain tumor classification.

\subsection{A proposed CNN Model for brain abnormality classification}

Brain tumor classification performs using CNN that is consists with several layers as displayed in the following figure 2. The convolutional layer is followed by an activation layer. We have used Rectified Linear Unit (ReLU) as an activation function. Moreover, batch normalization is used to make the network faster and stable. The addition of pooling layer after convolutional layer is a common pattern in CNN network. Here, max pooling layers are applied after some of the convolutional layers that calculate maximum value for each patch of a feature map. At last, flatten layer is applied to convert the data into one dimensional array to pass it as an input to the next layer. The fully connected layers are used for classification and the last fully connected layer outputs in three class. It gives " 1 " as an output for meningioma, " 2 " for glioma and " 3 " for pituitary tumor.

\begin{tabular}{|c|c|}
\hline Layer & Properties \\
\hline Image Input & $128 \times 128 \times 3$ \\
\hline Convolutional + ReLU & $\begin{array}{l}64 \text { Filters of size }(3 \times 3 \times 3) \text { with Padding }=\text { same, Rectified } \\
\text { Linear Unit }\end{array}$ \\
\hline $\begin{array}{l}\text { Convolutional }+ \text { ReLU }+ \\
\text { MaxPooling }\end{array}$ & $\begin{array}{l}64 \text { Filters of size }(3 \times 3 \times 3) \text { with Padding }=\text { same, Rectified } \\
\text { Linear Unit, } 2 \times 2 \text { max pooling with stride }[2 \text { 2 }]\end{array}$ \\
\hline $\begin{array}{l}\text { Convolutional + Batch } \\
\text { Normalization }+ \text { ReLU }\end{array}$ & $\begin{array}{l}64 \text { Filters of size }(3 \times 3 \times 3) \text { with Padding }=\text { same, Batch } \\
\text { Normalization, Rectified Linear Unit }\end{array}$ \\
\hline $\begin{array}{l}\text { Convolutional }+ \text { Batch } \\
\text { Normalization }+ \text { ReLU }+ \\
\text { MaxPooling }\end{array}$ & $\begin{array}{l}\text { 64 Filters of size }(3 \times 3 \times 3) \text { with Padding }=\text { same, } \quad \text { Batch } \\
\text { Normalization, Rectified Linear Unit, } 2 \times 2 \text { max pooling with } \\
\text { stride [2 2] }\end{array}$ \\
\hline $\begin{array}{l}\text { Convolutional + Batch } \\
\text { Normalization }+ \text { ReLU }\end{array}$ & $\begin{array}{l}64 \text { Filters of size }(3 \times 3 \times 3) \text { with Padding }=\text { same, } \\
\text { Batch Normalization, Rectified Linear Unit }\end{array}$ \\
\hline $\begin{array}{l}\text { Convolutional + Batch } \\
\text { Normalization }+ \text { ReLU }\end{array}$ & $\begin{array}{l}64 \text { Filters of size }(3 \times 3 \times 3) \text { with Padding }=\text { same, } \\
\text { Batch Normalization, Rectified Linear Unit }\end{array}$ \\
\hline $\begin{array}{l}\text { Convolutional }+ \text { Batch } \\
\text { Normalization }+ \text { ReLU }+ \\
\text { MaxPooling }\end{array}$ & $\begin{array}{l}64 \text { Filters of size }(3 \times 3 \times 3) \text { with Padding }=\text { same, } \\
\text { Batch Normalization, Rectified Linear Unit, } 2 \times 2 \text { max } \\
\text { pooling with stride [2 2] }\end{array}$ \\
\hline $\begin{array}{l}\text { Convolutional + Batch } \\
\text { Normalization + ReLU }\end{array}$ & $\begin{array}{l}64 \text { Filters of size }(3 \times 3 \times 3) \text { with Padding }=\text { same } \\
\text { Batch Normalization, Rectified Linear Unit }\end{array}$ \\
\hline $\begin{array}{l}\text { Convolutional + Batch } \\
\text { Normalization }+ \text { ReLU }\end{array}$ & $\begin{array}{l}128 \text { Filters of size }(3 \times 3 \times 3) \text { with Padding }=\text { same } \\
\text { Batch Normalization, Rectified Linear Unit }\end{array}$ \\
\hline $\begin{array}{l}\text { Convolutional + Batch } \\
\text { Normalization + ReLU }\end{array}$ & $\begin{array}{l}128 \text { Filters of size }(3 \times 3 \times 3) \text { with Padding }=\text { same } \\
\text { Batch Normalization, Rectified Linear Unit }\end{array}$ \\
\hline Flatten & Flatten into MLP \\
\hline $\begin{array}{l}\text { Fully Connected layer }+ \text { Batch } \\
\text { Normalization }+ \text { ReLU }\end{array}$ & $\begin{array}{l}256 \text { Neurons with Batch Normalization and Rectified Linear } \\
\text { Unit }\end{array}$ \\
\hline $\begin{array}{l}\text { Fully Connected layer + Batch } \\
\text { Normalization }+ \text { ReLU }\end{array}$ & $\begin{array}{l}256 \text { Neurons with Batch Normalization and Rectified Linear } \\
\text { Unit }\end{array}$ \\
\hline Fully Connected + Softmax & 3 Neurons in Last Layer for final output \\
\hline
\end{tabular}

Fig. 2. A Network Architecture of a proposed CNN Model for brain abnormality classification 


\section{Experimental Methodology}

The following figure 3 describes the experimental methods used in this research. Each deep learning model requires data for training. In this research, the dataset is acquired from Figshare data repository. The data is then converted from .mat file to .jpg file. There are three classes are available in dataset. Therefore, the images are organised into appropriate folders. Data augmentation is applied to the dataset to artificially enhance the size and diversity in the data. Then benchmark CNN models are identified that includes VGG16, VGG19, ResNET101, MobileNetV2 etc. For conducting experiment, we have used Keras and TensorFlow libraries for CNN model implementation [23]. The dataset is then after split into training, validation and testing. The ratio preserves is $80 \%, 10 \%$ and $10 \%$ respectively. To load the image, Keras provided ImageDataGenerator class that is used in the experiment. The models are built and compiled to start the training. The model is evaluated to obtain the results. Standard metrics like accuracy and loss are used for performance evaluation.

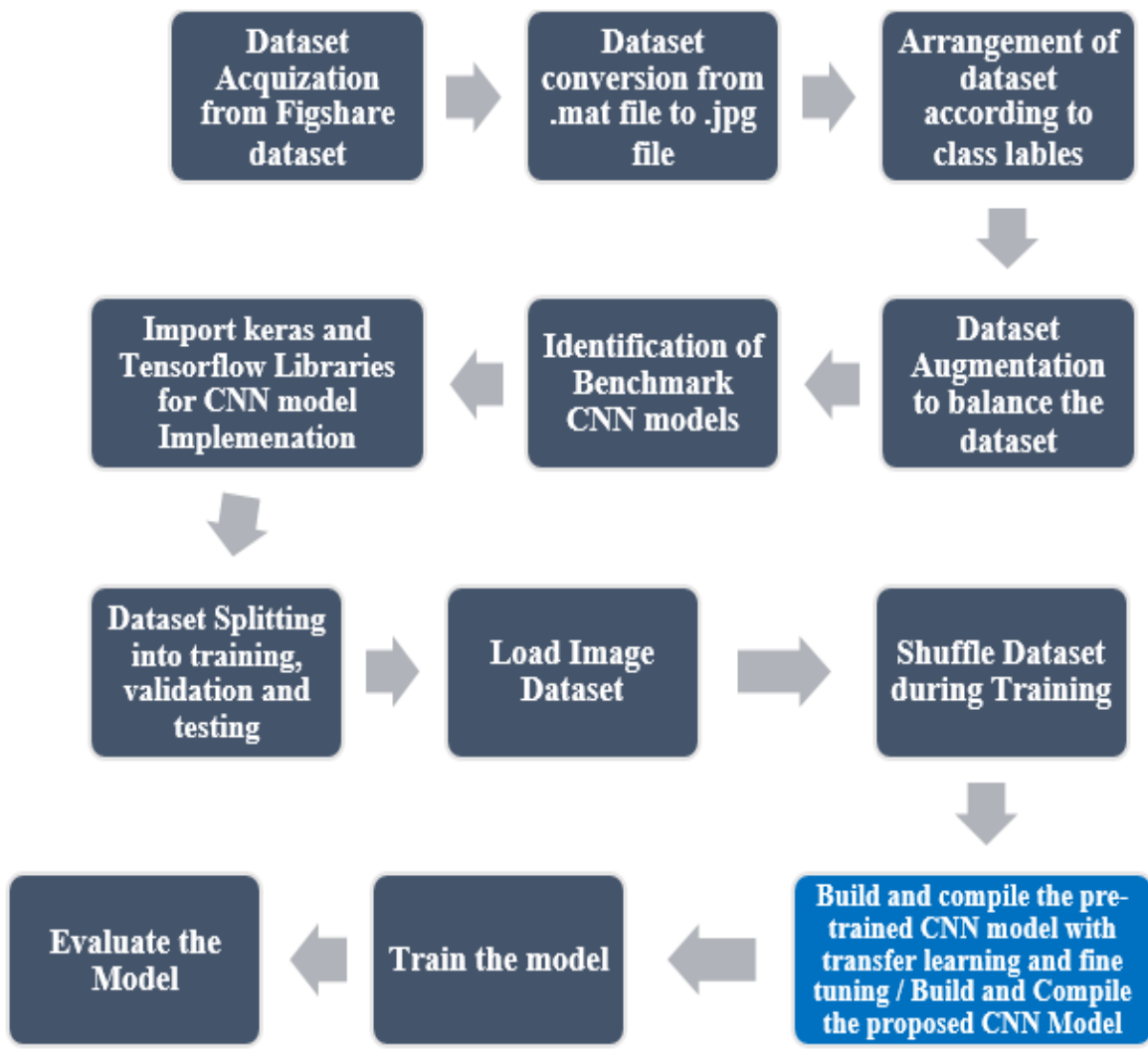

Fig. 3. An Experimental methodology for Training and Evaluation of CNN Models

\subsection{Hardware and Software}

For implementation, we have used Keras and TensorFlow deep learning libraries. Keras is a high level API that builds on the top of TensorFlow library. Moreover, Keras is specially designed for performing deep learning based applications and provides a convenient way to build CNN models. Keras is a high level API that uses Tensorflow or Theano as a backend. Keras is very simple and available with very good documentation and learning resources. TensorFlow is an open-source library that offers both high-level and low-level APIs.

For hardware and software support, a cloud platform namely Google Colab is used. Colab is a free Jupyter notebook environment that runs completely in the cloud. We have used Google Colab notebook and executed code on Google's GPU using Google's cloud servers. Colab supports a development environment in Python that makes easy to use other packages like Scikit, NumPy, Matplotlib etc.

\subsection{Hyperparameters and Network Training}

Hyperparameters are used to define the CNN structure and determines how the network is trained. The entire training process is governed by set of hyperparameters. It is required to set hyperparameters before training. There are various hyperparameters available including learning rate, optimizer, loss function, batch size, epoch etc [24]. The following are the details regarding the hyperparameters used in this experiment.

- Learning rate: Learning rate regulates the speed for learning weights by a model. A larger learning rate speeds up the learning but may the model not converged. A lower learning rate converges smoothly compared to a larger learning rate. A decaying rate of learning is preferred 
- Batch size: Batch size decides the number of samples passed in a model for a particular iteration. The typical sizes chosen are $32,64,128$, etc.

- Number of Steps: the number of steps defines the total number of training iterations required for the proposed object detection model. This number depends on the dataset's size and several other factors (including for how long the model does train). A training step is referred to as an update in one gradient. During one step, the amount of training samples assigns to batch size is processed.

- Activation function: Activation functions introduce non-linearity in the network and help to learn nonlinear prediction boundaries. ReLU, Sigmoid, Tanh, etc. are some of the most popular activation functions used in object detection models.

- Dropout rate: The dropout rate is used to solve the overfitting problem of the network. Dropout means dropping neurons of the network. They are chosen at random during the training process.

- Optimizer: Optimizer algorithms are used to calculate the optimal values for internal parameters (i.e., weights, bias, etc.). It helps to minimize the error function.

The following table 1 describes the values set for hyperparameters before training. The following values are obtained after tuning the hyperparameters to get optimum level of accuracy.

Table 1. Values sets for Hyperparameters

\begin{tabular}{|l|l|}
\hline Hyperparameter Name & Value \\
\hline Learning Rate & 0.001 \\
\hline Batch Size & 32 \\
\hline Training step or Iterations & $9652 / 32$ (Total training samples/batch size) \\
\hline Epoch & 20 \\
\hline Optimizer & SGD \\
\hline Loss Function & Categorical Cross-entropy \\
\hline Dropout rate & 0.2 \\
\hline Epoch & 20 \\
\hline
\end{tabular}

\subsection{Loss Functions}

There are two performance parameters most often used to measure the performance of CNN model i.e. accuracy and loss. The aim of a good training is not only about increase the accuracy but also to decrease the loss. This can be achieved by optimizing the weights of CNN model. Loss is a function that measures an error occurred in prediction by a model [25]. There are two functions generally used to measure loss i.e. mean square error and categorical cross entropy. To determine the performance of a CNN model, accuracy and loss can be graphically described as shown in figure 4. The loss and accuracy are measured at two stages; training and validation. In following figure 4 , training and validation accuracy and losses are going parallel and closely to each other. This shows that model is trained adequately without underfitting and overfitting.
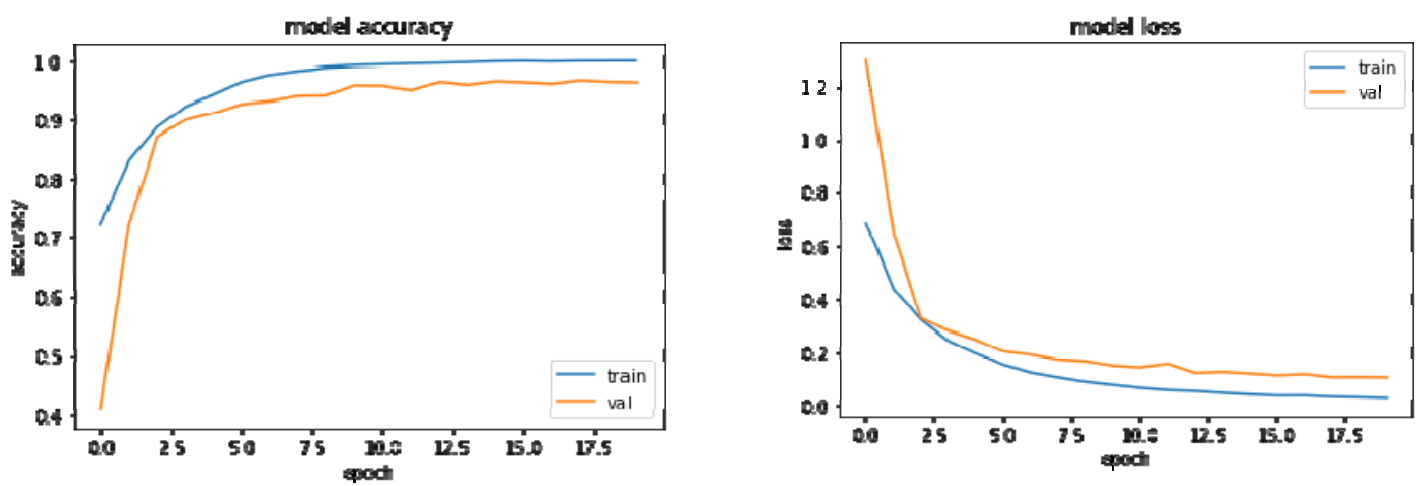

Fig. 4. Accuracy and Loss for the Proposed Model 


\section{Results and Discussion}

The following table 2 shows the total number of parameters, trainable parameters and non-trainable parameters contain by a CNN model. Most of the benchmark models are having large number of parameters to learn as they having very deep network architecture. Whereas, from table 2, it has found that, the proposed CNN model is having lowest number of parameters without compromising accuracy.

Table 2. Parameters Learned by CNN Models

\begin{tabular}{|l|l|l|l|l|l|l|l|}
\hline & VGG16 & VGG19 & $\begin{array}{l}\text { MobileNet } \\
\text { V2 }\end{array}$ & ResNet50 & ResNet101 & $\begin{array}{l}\text { Densenet } \\
\mathbf{1 2 1}\end{array}$ & $\begin{array}{l}\text { Proposed } \\
\text { CNN }\end{array}$ \\
\hline $\begin{array}{l}\text { Total } \\
\text { params }\end{array}$ & $21,204,035$ & $26,513,731$ & $18,381,123$ & $49,321,731$ & $68,383,491$ & $19,949,379$ & $2,683,075$ \\
\hline $\begin{array}{l}\text { Trainable } \\
\text { params }\end{array}$ & $6,489,347$ & $6,489,347$ & $16,157,251$ & $25,802,371$ & $25,854,595$ & $12,995,523$ & $2,664,387$ \\
\hline $\begin{array}{l}\text { Non- } \\
\text { trainable } \\
\text { params }\end{array}$ & $14,714,688$ & $20,024,384$ & $2,223,872$ & $23,519,360$ & $42,528,896$ & $6,953,856$ & 18,688 \\
\hline
\end{tabular}

The following table 3 shows the accuracy and loss values for different CNN models. The values for accuracy and loss are provided for training, validation and testing. From the results, it can be found that, VGG16 and VGG19 obtained the lowest accuracy and highest loss. MobileNetV2 performs better than VGG16 and VGG19. Both of the variants for ResNet i.e. ResNet50 and ResNet101 work well including Densenet121. Among all, the proposed CNN model outperforms with highest training, validation and testing accuracy i.e. 0.9985, 0.9637 and 0.9670. Moreover, the values for losses are also lowest i.e. $0.0246,0.1018$ and 0.1038 respectively. Along with the optimum values of accuracy and loss, the time taken to train the entire model is significantly less for the proposed CNN model.

Table 3. Accuracy and Loss for CNN Models

\begin{tabular}{|l|l|l|l|l|l|l|l|}
\hline Parameters & VGG16 & VGG19 & $\begin{array}{l}\text { MobileNet } \\
\text { V2 }\end{array}$ & ResNet50 & ResNet101 & $\begin{array}{l}\text { Densenet } \\
\mathbf{1 2 1}\end{array}$ & $\begin{array}{l}\text { Proposed } \\
\text { CNN }\end{array}$ \\
\hline $\begin{array}{l}\text { Training } \\
\text { accuracy }\end{array}$ & 0.8877 & 0.8625 & 0.9762 & 0.9925 & 0.9902 & 0.9910 & 0.9985 \\
\hline Training Loss & 0.2838 & 0.3426 & 0.0647 & 0.0254 & 0.0319 & 0.0283 & 0.0246 \\
\hline $\begin{array}{l}\text { Validation } \\
\text { Accuracy }\end{array}$ & 0.8730 & 0.8606 & 0.8994 & 0.9417 & 0.9506 & 0.9633 & 0.9637 \\
\hline Validation Loss & 0.2159 & 0.3207 & 0.2477 & 0.1687 & 0.3082 & 0.1418 & 0.1018 \\
\hline $\begin{array}{l}\text { Testing } \\
\text { Accuracy }\end{array}$ & 0.8657 & 0.8690 & 0.9062 & 0.9594 & 0.9552 & 0.9670 & 0.9670 \\
\hline $\begin{array}{l}\text { Testing Loss } \\
0.4759\end{array}$ & 0.3215 & 0.1839 & 0.1166 & 0.1248 & 0.1620 & 0.1038 \\
\hline $\begin{array}{l}\text { Time taken to } \\
\text { Train the Model }\end{array}$ & $0: 33: 53$. & $\begin{array}{l}0: 32: 54 \\
.186228\end{array}$ & $\begin{array}{l}0: 31: 25.39 \\
9036\end{array}$ & $\begin{array}{l}0: 47: 29.71 \\
7618\end{array}$ & $\begin{array}{l}1: 14: 41.84 \\
9550\end{array}$ & $\begin{array}{l}1: 15: 42.38 \\
6142\end{array}$ & $\begin{array}{l}0: 14: 18.36 \\
6423\end{array}$ \\
\hline
\end{tabular}

\section{Conclusion}

In this paper, we have developed and proposed a convolutional neural network (CNN) model for multi-class classification of brain abnormality. Brain tumor is considered one of the major brain abnormalities and therefore, the model is applied and experimented on brain tumor dataset. Several benchmark CNN models that include VGG16, VGG19, MobileNetV2, ResNet50, ResNet101 and DenseNet121 are applied and evaluated for performance comparison. By seeing the results, it can be found that, the proposed CNN model outperforms among other $\mathrm{CNN}$ models. It consumes less parameters and less time for training. From the graphs of accuracy and loss, it is observed that the proposed model converge optimally as compared to other CNN models. Moreover, the highest accuracy and lowest loss are also obtained from the proposed model. 


\section{References}

[1] Villanueva-Meyer, JE.; Mabray, MC.; Cha S. (2017): Current Clinical Brain Tumor Imaging. Neurosurgery, 81(3), pp. 397-415.

[2] Zacharaki, EI.; Wang, S.; Chawla, S; et al. (2009): Classification of brain tumor type and grade using MRI texture and shape in a machine learning scheme. Magn Reson Med., 62(6), pp.1609-1618.

[3] Zhao, J.; Meng, Z.; Wei, L.; Sun, C.; Zou, Q.; \& Su, R. (2019): Supervised Brain Tumor Segmentation Based on Gradient and Context-Sensitive Features. Frontiers in neuroscience, 13(144).

[4] Brain Tumor: Statistics, Cancer.Net Editorial Board, 11/2017 (Accessed on 17th January 2019)

[5] Kavitha, A.R. \& Chellamuthu, Chinna. (2018): Advanced Brain Tumour Segmentation from MRI Images. 10.5772/intechopen.71416.

[6] Patel, S. (2020): A Comprehensive Analysis of Convolutional Neural Network Models. International Journal of Advanced Science and Technology, 29(04), pp. $771-777$.

[7] Patel, S.; Patel, A. (2018): Deep Leaning Architectures and its Applications: A Survey. International Journal of Computer Sciences and Engineering, 6(6), pp. 1177-1183.

[8] Li, Ming \& Kuang; Lishan \& Xu; Shuhua \& Sha; Zhanguo. (2019). Brain Tumor Detection Based on Multimodal Information Fusion and Convolutional Neural Network. IEEE Access, 7, pp. 180134-180146.

[9] T., Hossain; F., S., Shishir; M., Ashraf; M., A., Al Nasim; F. Muhammad Shah: "Brain Tumor Detection Using Convolutional Neural Network," 2019 1st International Conference on Advances in Science, Engineering and Robotics Technology (ICASERT), Dhaka, Bangladesh, 2019, pp. 1-6.

[10] H. UCUZAL; A. G. İ. BALIKÇI ÇIÇEK,; A. G. A. K. ARSLAN; and C. ÇOLAK: "A Web-Based Application for Identifying Objects In Images: Object Recognition Software," 2019 3rd International Symposium on Multidisciplinary Studies and Innovative Technologies (ISMSIT), Ankara, Turkey, 2019, pp. 1-5, doi: 10.1109/ISMSIT.2019.8932735.

[11] Zahra, Sobhaninia; Safiyeh, Rezaei; Alireza, Noroozi; Mehdi, Ahmadi; Hamidreza, Zarrabi; Nader, Karimi; Ali, Emami; Shadrokh, Samavi.(2018): Brain Tumor Segmentation Using Deep Learning by Type Specific Sorting of Images, arXiv:1809.07786.

[12] S., Zhou; D., Nie; E., Adeli; J., Yin; J., Lian; D., Shen. (2020): High-Resolution Encoder-Decoder Networks for Low-Contrast Medical Image Segmentation, in IEEE Transactions on Image Processing, vol. 29, pp. 461-475

[13] Pereira S,; Pinto A,; Alves V,; Silva CA.(2016): Brain Tumor Segmentation Using Convolutional Neural Networks in MRI Images. IEEE Trans Med Imaging. 35(5), pp. 1240-1251.

[14] Alqazzaz, S.; Sun, X.; Yang, X. et al. (2019): Automated brain tumor segmentation on multi-modal MR image using SegNet. Comp. Visual Media 5, 209-219

[15] S. Hussain,; S. M. Anwar; and M. Majid, "Brain tumor segmentation using cascaded deep convolutional neural network," 2017 39th Annual International Conference of the IEEE Engineering in Medicine and Biology Society (EMBC), Seogwipo, 2017, pp. 1998-2001.

[16] fighshare data

repository, https://figshare.com/articles/brain_tumor_dataset/1512427\#: :text=This\%20brain $\% 20$ tumor\%20dataset $\% 20$ contains,be $\% 20$ found $\% 20$ in $\% 20$ readme $\% 20$ file.

[17] A. Mikołajczyk; M. Grochowski, "Data augmentation for improving deep learning in image classification problem," 2018 International Interdisciplinary PhD Workshop (IIPhDW), Swinoujście, 2018, pp. 117-122.

[18] Ganatra, N.; \& Patel, A. (2018). A Comprehensive Study of Deep Learning Architectures, Applications and Tools. International Journal of Computer Sciences and Engineering. vol. 6. pp. 701-705.

[19] Ganatra, N.; and Patel, A. (2020). A Multiclass Plant Leaf Disease Detection using Image Processing and Machine Learning Techniques. International Journal on Emerging Technologies, 11(2), pp. 1082-1086.

[20] Simonyan, K.; Zisserman, A. (2015). Very Deep Convolutional Networks for Large-Scale Image Recognition. CoRR, abs/1409.1556.

[21] Andrew G. Howard; Menglong Zhu; Bo Chen, Dmitry Kalenichenko; Weijun Wang; Tobias Weyand; Marco Andreetto; Hartwig Adam; MobileNets: Efficient Convolutional Neural Networks for Mobile Vision Applications, arXiv:1704.04861.

[22] Liu, Bing; Liu, Qiao; Zhu, Zhengyu; Zhang, Taiping; Yang, Yong. (2019). MSST-ResNet: Deep multi-scale spatiotemporal features for robust visual object tracking. pp.235-252.

[23] TensorFlow, https://www.tensorflow.org/

[24] Mboga, Nicholus; Persello, Claudio; Bergado, John Ray; Stein, Alfred. (2017). Detection of Informal Settlements from VHR Images Using Convolutional Neural Networks. Remote Sensing. 9(1106), 10.3390/rs9111106.

[25] Galván Tejada, Carlos \& Zanella Calzada, Laura \& García-Domínguez, Antonio \& Magallanes-Quintanar, Rafael \& Luna-Garcia, Huizilopoztli \& Celaya Padilla, Jose \& Galván Tejada, Jorge \& Vélez-Rodríguez, Alberto \& Gamboa-Rosales, Hamurabi.: (2020). Estimation of Indoor Location Through Magnetic Field Data: An Approach Based On Convolutional Neural Networks. ISPRS International Journal of Geo-Information, 9(226) 10.3390/ijgi9040226. 